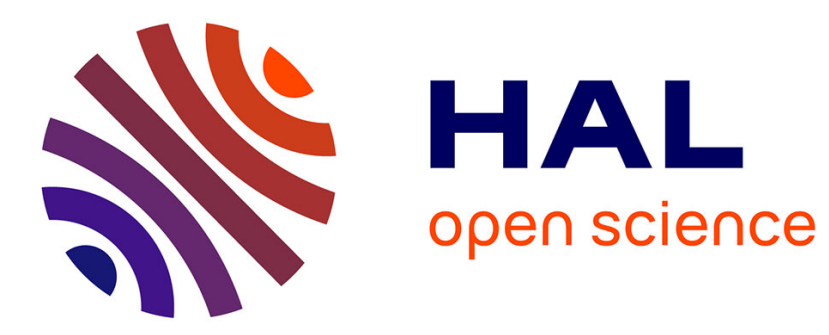

\title{
La grotte d'Alisadr un témoin exceptionnel de l'évolution morphologique du Zagros (Iran)
}

\author{
Dominique Dumas
}

\section{To cite this version:}

Dominique Dumas. La grotte d'Alisadr un témoin exceptionnel de l'évolution morphologique du Zagros (Iran). KARSTOLOGIA, 2004, 43 (1), pp.1-8. halshs-00324627

\section{HAL Id: halshs-00324627 https://shs.hal.science/halshs-00324627}

Submitted on 12 Feb 2010

HAL is a multi-disciplinary open access archive for the deposit and dissemination of scientific research documents, whether they are published or not. The documents may come from teaching and research institutions in France or abroad, or from public or private research centers.
L'archive ouverte pluridisciplinaire $\mathbf{H A L}$, est destinée au dépôt et à la diffusion de documents scientifiques de niveau recherche, publiés ou non, émanant des établissements d'enseignement et de recherche français ou étrangers, des laboratoires publics ou privés. 


\title{
La grotte d'Alisadr un témoin exceptionnel de l'évolution morphologique du Zagros (Iran).
}

\section{Cave of Alisadr : a geomorphological site of outstanding interect in Zagros mountains of Iran}

D. Dumas, 2004. Karstologia, n43, pp. 1-8.

Résumé : Située sur la bordure orientale du Zagros, la grotte touristique d'Alisadr est actuellement la plus grande cavité souterraine connue d'Iran. L'essentiel du réseau souterrain est noyé en permanence et présente sur ses parois un admirable étagement de niveaux concrétionnés qui témoignent de paléoniveaux de la nappe karstique. L'endokarst a conservé de nombreux témoins et indices paléoenvironnementaux qui permettent retracer son évolution. Par ailleurs, les datations effectuées sur trois des niveaux les plus remarquables, ainsi que sur une ancienne coulée volcanique perchée, située à quelques kilomètres de la grotte, offrent l'occasion de positionner chronologiquement cette évolution et d'estimer un peu mieux la part des héritages antéquaternaires sur les paysages de cette région. Entre autres, l'inexistence d'une érosion glaciaire, la dissolution spécifique actuelle, proche de $3 \mathrm{~mm} / \mathrm{millénaire,} \mathrm{les}$ processus de bréchification militent pour une évolution assez modeste des morphologies exokarstiques et endokarstiques au cours du Quaternaire.

Mots clés : karst, karstogenèse, spéléogenèse, hydrogéologie, remplissage endokarstique, morphologie zndokarstique, grotte d'Alisadr, Zagros, Iran

\begin{abstract}
The tourist cave of Alisadr, located on the eastern boundaries of the Zagros Mountains, is biggest subsurface cave visited in Iran. Most part of the karstic underground galleries are permanently filled with water: on the sides of the galleries former water table levels are indicated by numerous calcareous sinters. The sub-surface karst has preserved numerous relics and paleoenvironmental residual deposits which show the geomorphological karstic development. Datating of three conspicuous calcareous levels in the cave and that of the surface basaltic mesa, to be established a few kilometers from the cave enable a chronology the stages of karstic evolution. The place of prequaternary vestiges in the landscapes of this country is also determined. For example, no typical landform of glacial erosion has been identified. The current karstic denudation rate is about $3 \mathrm{~mm} / \mathrm{Ky}$. The geomorphological evolution of surface and sub-surface landforms during the quaternary era is shown and deduced from the processes which have led to breccias formations in calcareous rocks.
\end{abstract}

Key words: karst, kartogenesis, speleogenesis, hydrogeology, endokarstic fillings, endokarstic morphology, Alisadr cave, Zagros, Iran.

\section{INTRODUCTION}

Après les ruines de Persépolis, la grotte d'Alisadr est le site touristique le plus visité d'Iran. Il n'est d'ailleurs pas impossible qu'en nombre d'entrées, elle représente le première site touristique iranien (Dumas et al., 1994). Située à $60 \mathrm{~km}$ au NNW d'Hamadan, la grotte d'Alisadr se place dans le domaine métamorphique du Zagros, en bordure septentrionale de la chaîne du Zagros (figure 1). Elle s'ouvre à environ $2000 \mathrm{~m}$ d'altitude à l'extrémité nord d'une série de buttes coiffées par des calcaires métamorphisés culminant à $2500 \mathrm{~m}$ et alignées grossièrement selon un axe nord-sud qui forment les monts de Sarighayeh (figure 2, photos 1 et 2).

Cette étude se propose de retracer la genèse de cette grande cavité en caractérisant les étapes successives de son évolution. La genèse de ce grand réseau est probablement ancienne et comporte différentes phases morphogéniques. Ainsi, l'étude de la grotte d'Alisadr permet de compléter le schéma d'évolution générale de la chaîne du Zagros. Pour cela, la part de l'héritage géomorphologique et le legs des paléoenvironnements successifs doivent être décryptés le plus minutieusement possible.

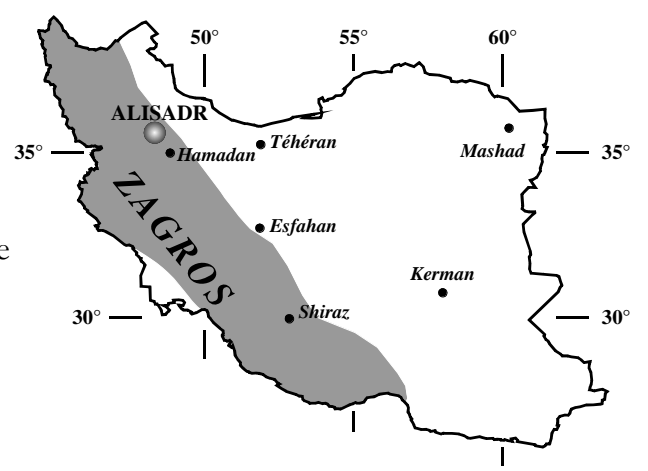




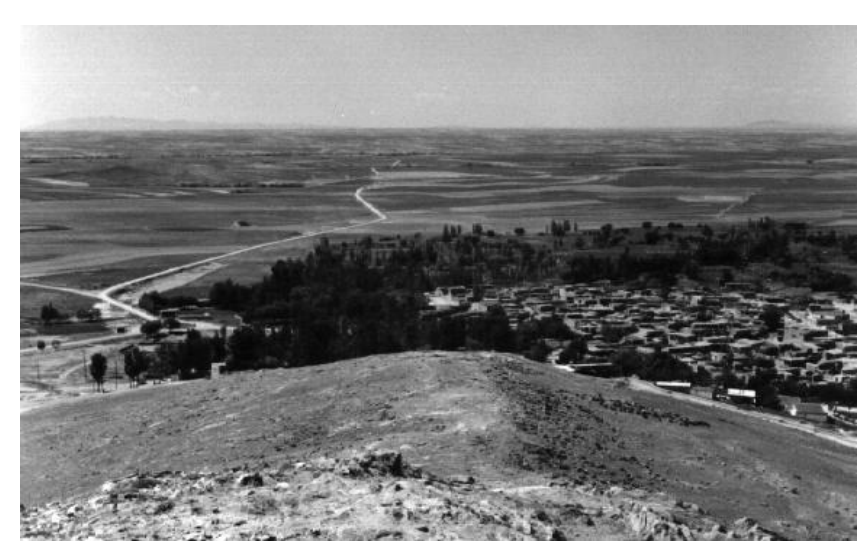

Photo 1. Depuis le sommet de la colline, vue sur la plaine d'Alisadr développée dans les schistes jurassiques et du village d'Alisadr

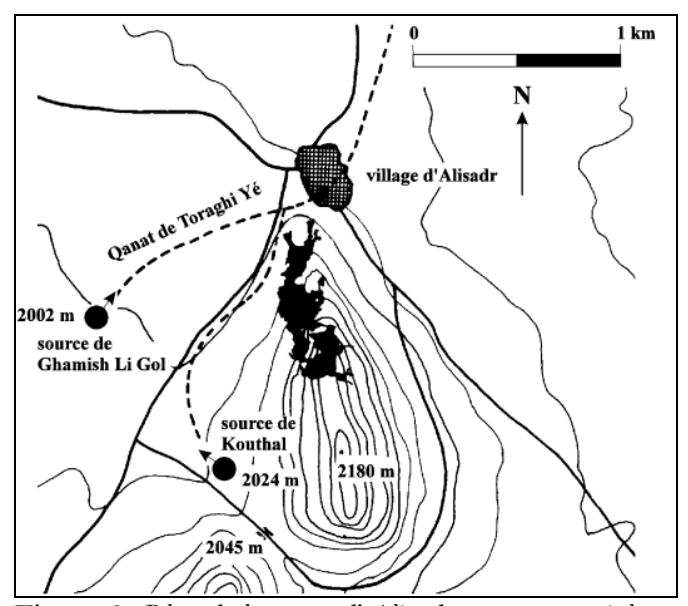

Figure 2. Plan de la grotte d'Alisadr par rapport à la topographie de surface

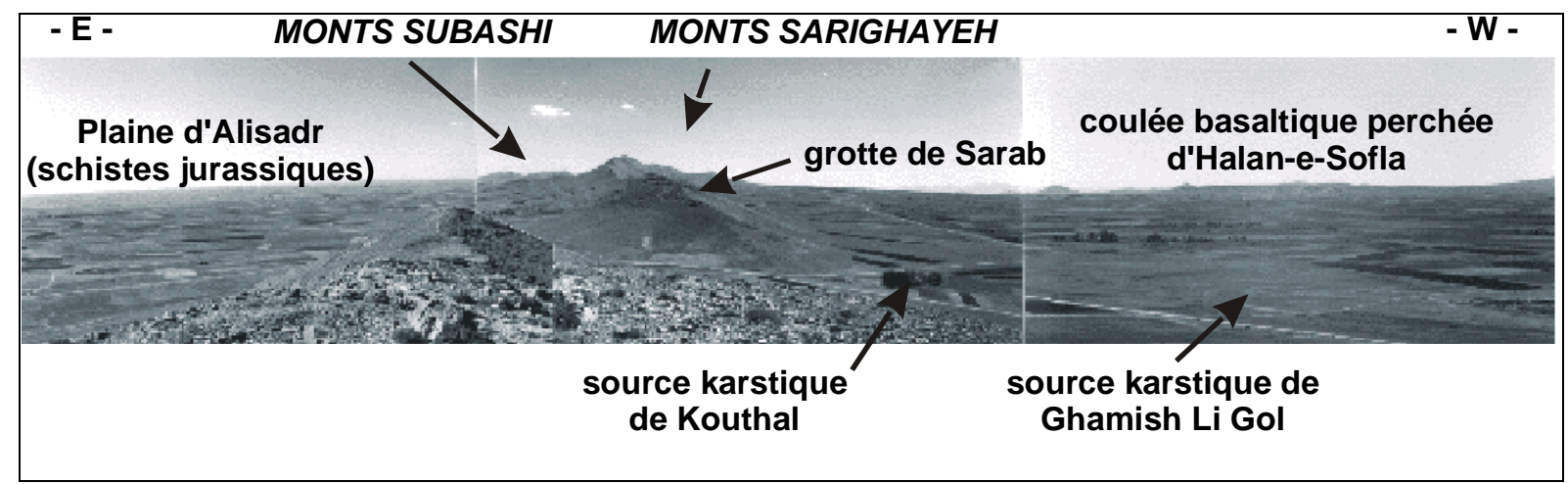

Photo 2. Panorama de la région d'Alisadr depuis le sommet de la butte calcaire où se développe la grotte d'Alisadr

\section{LE CONTEXTE GEOLOGIQUE}

Ce domaine est caractérisé par de vastes plaines, placées à $2000 \mathrm{~m}$ d'altitude, et dominées par des affleurements schisteux d'aspect lustré et de couleur noire ou gris-vert. Cette série, fortement plissée, reste exceptionnellement homogène et monotone puisqu'on la retrouve sur de très grandes étendues, de Golpaygan jusqu'à Esfahan (Braud, 1987). Le style plissé de cette formation se poursuit sur les lambeaux de calcaires qui la coiffent (figure 3). Les bombements anticlinaux ont favorisé leur décapage ; à l'inverse les creux structuraux ont favorisé leur conservation. La grotte d'Alisadr s'ouvre et se développe dans ces calcaires métamorphisés du Jurassique, et la formation schisteuse sous-jacente représente un niveau imperméable qui bloque le développement vertical de l'endokarst.

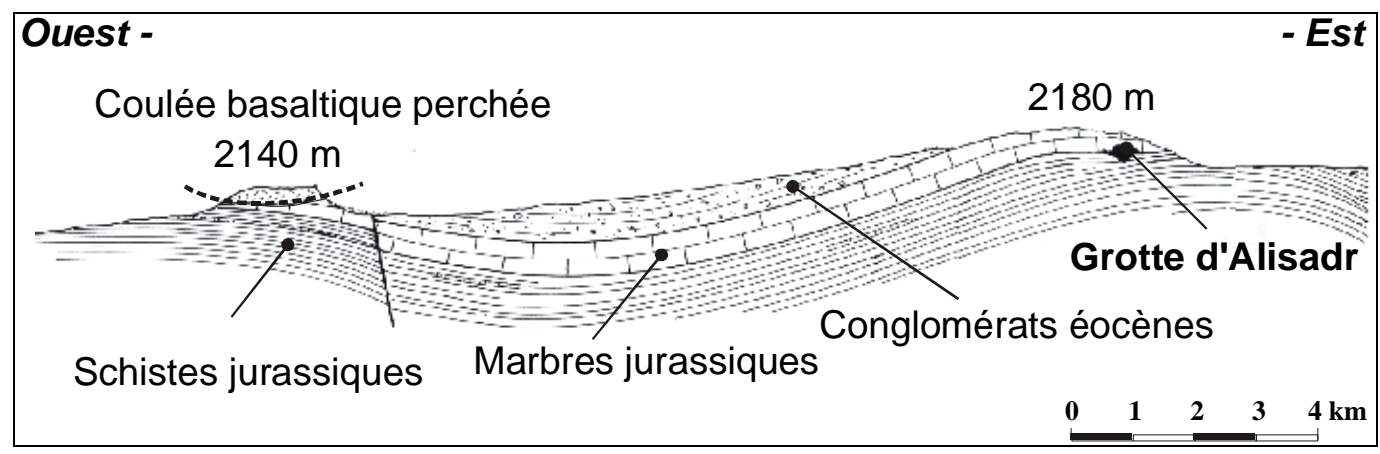

Figure 3. Coupe schématique de la plaine d'Alisadr (échelle des hauteurs exagérée). 
L'endokarst très développé des Monts Sarighayeh s'explique notamment par une forte fracturation des séries carbonatées mais également par la recristallisation des cristaux microsparitiques de ces calcaires. Cependant, la dissolution est freinée par un taux d'insolubles relativement élevé, qui en moyenne atteint 20\% de la roche (photo 3). C'est sans doute cette caractéristique qui limite en surface le développement de lapiés.

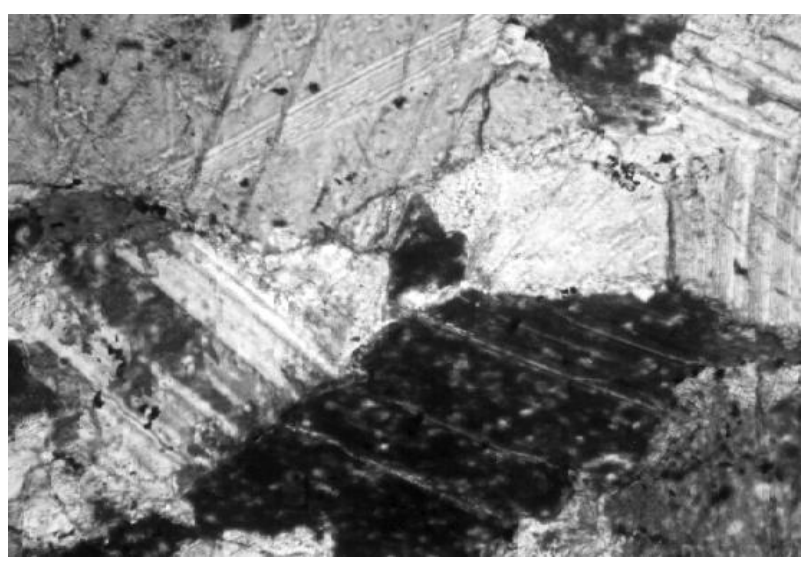

Photo 3. L'analyse microscopique d'échantillons de marbre montre un ensemble de cristaux microsparitiques. Sous l'effet de pression, une dissolution sélective apparaît entre les grains. Les carbonates montrent une imbrication et une suture grain à grain liée à la phase de métamorphisme régionale. On observe une accumulation préférentielle le long de veines des éléments insolubes, comme les argiles et les quartz. (longueur $=0,66 \mathrm{~mm}$ ). cutanes argileuses ; micro-grès à éléments métamorphiques, schistes, grains quartzitiques et de quartz, phyllites, roches silicatées et morceaux de roches carbonatées du Mésozoïque. L'absence d'éléments tertiaires remaniés et la diagenèse évoluée, avec une cimentation carbonatée très compacte, semblent indiquer un âge éocène.

Les cartes géologiques des régions avoisinantes (de Hamadan, Golpaygan et Kabudar Ahang) semblent confirmer cette hypothèse. La description des conglomérats éocènes, présents localement sur ces différents secteurs, montre une bonne correspondance avec ceux observés dans la région d'Alisadr. Ces différents arguments militent pour rattacher cette couverture détritique à la formation éocène de "Fajan". Elle est d'ailleurs probablement consécutive du soulèvement régional de la fin du Crétacé (Ricou, 1976 ; Braud 1987 ; Dumas, 1998).

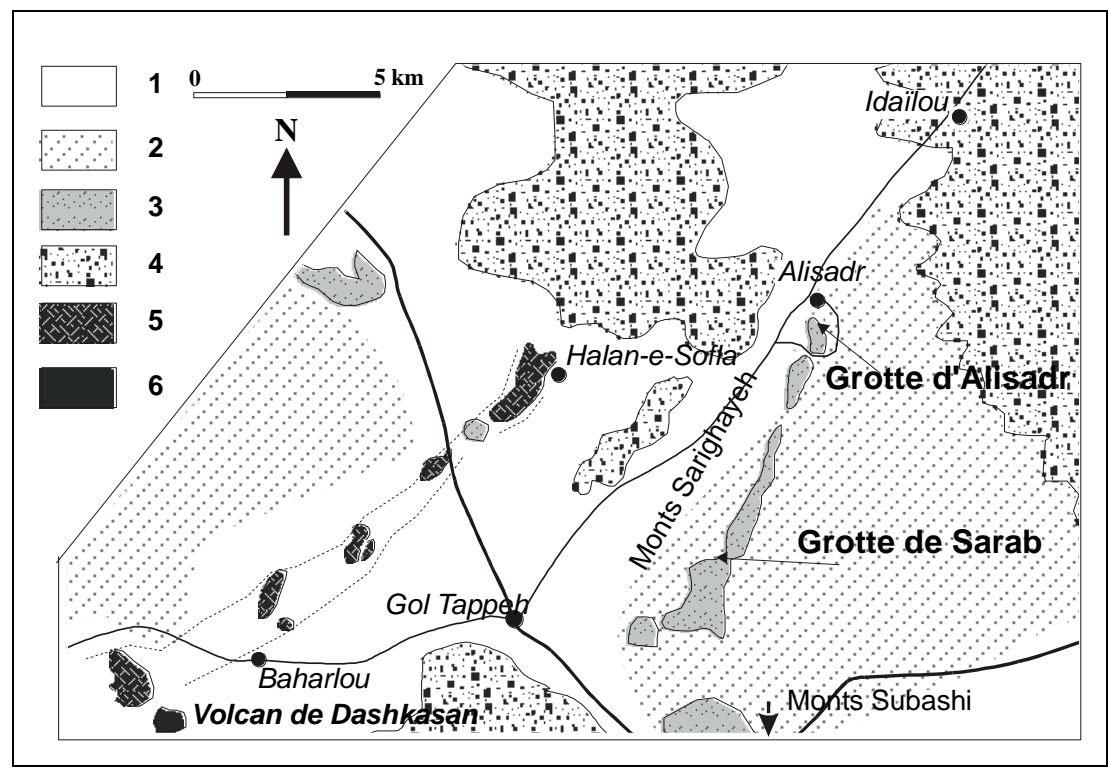

Figure 4. Carte géologique simplifiée de la région d'Alisadr à partir de relevés de terrains (fragmentaires) et de photographies aériennes au 1/50.000ème.

1 : Dépôts du Quaternaire ; 2 : Schistes jurassiques ; 3 : Marbres jurassiques ; 4 : Conglomérats éocènes ; 5 : Coulées volcaniques basaltiques ; 6 : Pyroclastes à trachytes. 


\section{LE KARST DES MONTS SARIGHAYEH}

\section{A. La grottes d'Alisadr}

L'ensemble de la cavité d'Alisadr se développe selon une direction méridienne qui correspond à une gouttière synclinale d'axe nord-sud inscrite au sein d'un large bombement anticlinal dans les séries schisteuses du Jurassique. L'essentiel du réseau souterrain se place au contact des schistes imperméables et des marbres karstifiables sous la forme d'un dédale de galeries horizontales (figure 5). Une grande partie du réseau souterrain montre une spéléogenèse de type noyé avec des protubérances ou des pendants de plafond, des cloches emboîtées à la voûte et des parois intensément corrodées.

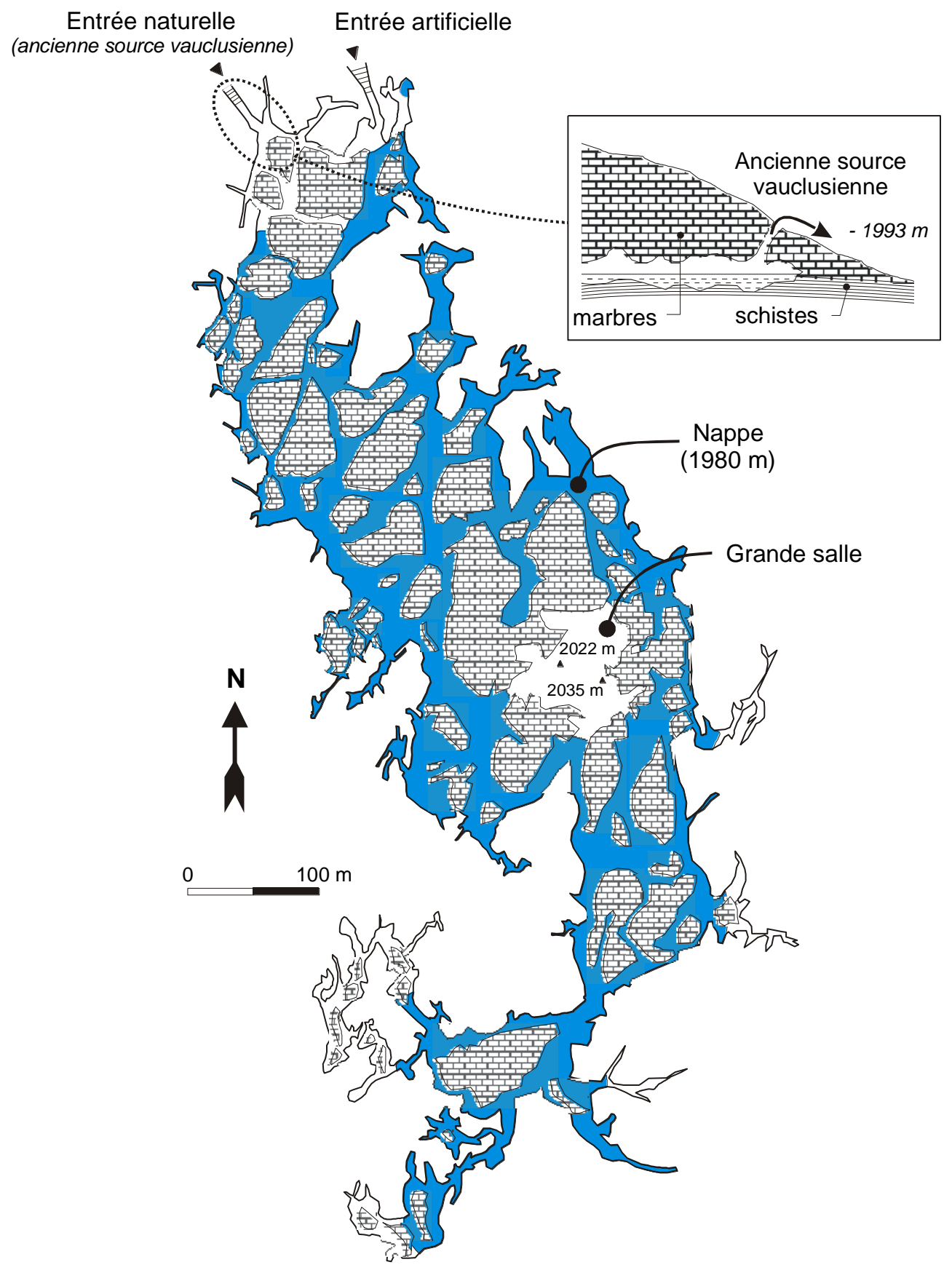

Figure 5. Plan de la cavité d'Alisadr

(à partir d'un relevé topométrique effectué en 2000 par la compagnie touristique d'Alisadr) 
Aujourd'hui, le réseau souterrain est à plus de $80 \%$ noyé en permanence, le battement saisonnier de la nappe, d'environ $1 \mathrm{~m}$, ne modifie pas cet état. Le plan général du réseau avec un entrelacs de galeries, dont la longueur cumulée dépasse probablement $5 \mathrm{~km}$, montre qu'il s'agit d'une cavité de type "delta souterrain" (figure 5).

La nappe phréatique est située à $1980 \mathrm{~m}$ d'altitude (figure 5). On y accède par une entrée naturelle, dont l'orifice, placé à $13 \mathrm{~m}$ au-dessus de la nappe, se prolonge par une galerie vertical subcirculaire. La morphologie et les formes pariétales de type noyé de ce conduit témoignent qu'il s'agissait d'une ancienne source vauclusienne. Elle était active lorsque les Monts de Sarigayeh, voire même les Monts de Subashi (plus au sud), étaient connectés hydrologiquement.

Le réseau comporte au centre de la cavité une salle souterraine de grandes dimensions : $120 \mathrm{~m}$ de longueur dans le sens NNW-SSE et environ $80 \mathrm{~m}$ de large dans le sens N-S (figures 5). L'éboulement progressif du plafond et des parois a engendré deux buttes distinctes dans cette salle ; l'une culmine à $2035 \mathrm{~m}$ et l'autre plus large et plus empâtée à $2022 \mathrm{~m}$.

\section{B. La grotte Sarab}

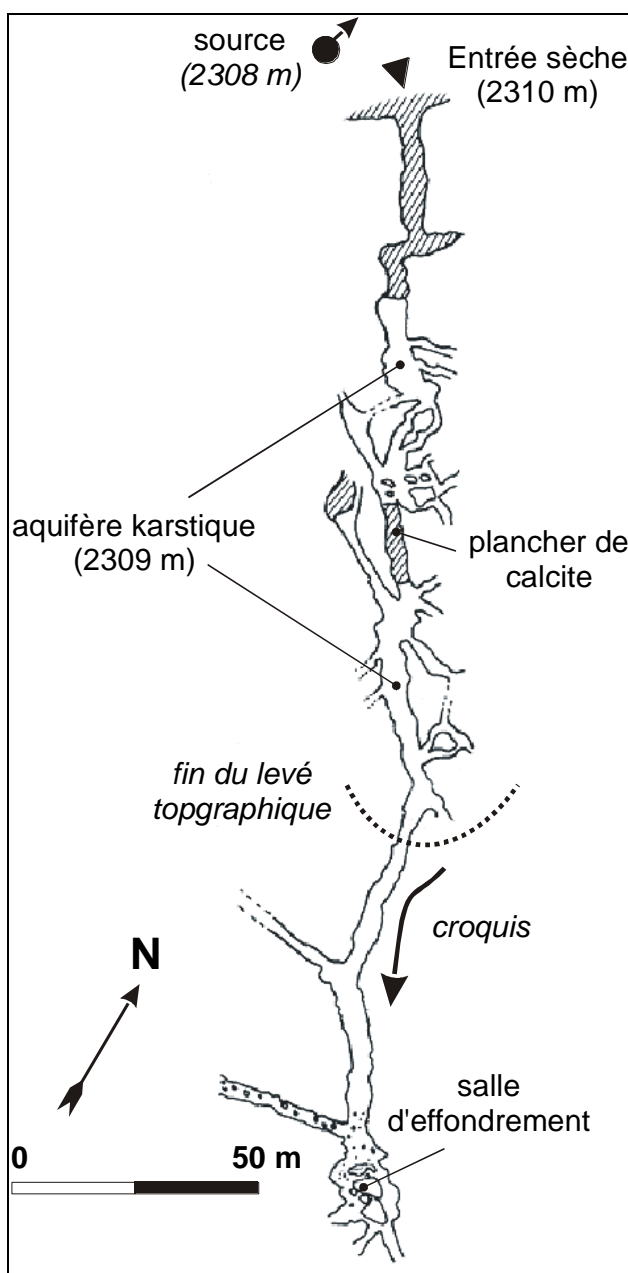

Figure 6. Plan de la grotte de Sarab

(Topographie : expédition Zagros 1996 ; Dessin : D

Dumas)
Située à $10 \mathrm{~km}$ plus au sud de la cavité d'Alisadr, la grotte de Sarab présente un développement important avec plus de $500 \mathrm{~m}$ de galeries reconnues et topographiées (figure 6). L"ensemble du réseau est semi-noyée avec une profondeur d'eau dépassant parfois $2 \mathrm{~m}$. Contrairement à la grotte d'Alisadr, cette cavité ne présente pas une morphologie de delta souterrain et l'on suit facilement, sans trop pouvoir s'égarer, une galerie principale de direction subméridienne.

La morphologie d'ensemble de la grotte de Sarab semble montrer qu'elle a été un chaînon hydrologique au cœur de l'endokarst des Monts Sarighayeh. L'eau circulait dans les conduits karstiques de la grotte de Sarab, collectée en amont par les collines situées plus au sud et sans doute même par l'endokarst des Monts Subashi, pour aboutir très vraisemblablement dans le delta souterrain d'Alisadr et sortir par sa source vauclusienne aujourd'hui sénile. En effet, la genèse de la cavité n'est certainement pas uniquement liée aux variations piézométriques de la nappe phréatique, car de nombreux conduits portent la trace d'un creusement fluviatile en régime noyé avec de nombreux conduits circulaires souvent tapissés de cupules d'érosion ou de "vagues d'érosion" (Goodchild et al., 1971 ; Lismonde et al., 1987).

\section{Le karst des Monts Sarighayeh et ses indices paléoenvironnentaux}

Les Monts Sarigayeh et la cavité d'Alisadr ont conservé différents témoins d'anciennes phases morphogéniques. La conservation de ces éléments permet de mieux cerner l'évolution géomorphologique de cette région. 


\section{- Bréchification des marbres}

On retrouve sur les buttes calcaires des traces d'une paléokarstification avec une infiltration de carbonate de fer. La présence de sidérose, avec des minéraux de fer spathiques, dans les sols des collines des Monts Sarighayeh est nettement visible sur lames minces (photo 4). Par ailleurs, l'analyse aux rayons X de ces sols montre la présence de goethite (Dumas, 1998). La présence du fer est peut être d'origine hydrothermale et liée aux intrusions magmatiques et au volcanisme régional. L'association de ces sidéroses à des brèches minéralisées est généralement la trace d'une ancienne karstification de caractère syngénétique (Nicod, 1996). La grotte d'Alisadr a recoupé une partie de ces brèches puisqu'on les retrouve dans l'endokarst tout comme sur l'exokarst.

Ce processus de dissolution et de bréchification a ainsi préparé et facilité le travail de l'érosion. Dans l'endokarst, ces processus de bréchification entraînent des effets mécaniques, avec des appels au vide qui ont favorisé le développement des galeries souterraines. Sur l'exokarst, les restes d'une ancienne cuirasse ferrugineuse altérée et les processus de bréchification sont les témoins d'une altération karstique ancienne.

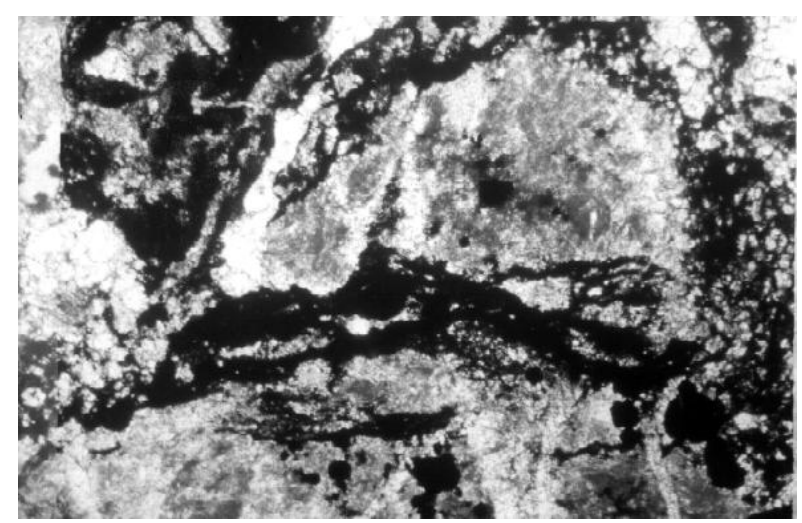

Photo 4. L'échantillon (L.P.) montre les calcaires marbrifiés fortement altérés par un processus de bréchification (longueur $=$ $4,25 \mathrm{~mm}$

\section{- Remplissage conglomératique}

Non loin de l'entrée de la cavité, dans une galerie semi-noyée, on observe la trace d'un ancien remplissage détritique relativement bien conservé par une induration calcitique et dont une partie a été entaillée pour l'aménagement de la cavité (photo 5). L'observation à l'échelle macroscopique de ce témoin d'un remplissage fluviatile montre qu'il est constitué de nombreux galets d'origine lithologique variée et dont certains peuvent atteindre un diamètre de $20 \mathrm{~cm}$. Tous ces éléments sont fortement altérés. Les fantômes de galets se fragmentent avec une lame de couteau. Cet état d'altération des différents éléments suggère un âge relativement ancien. La texture de ce conglomérat rappele les conglomérats éocènes que l'on retrouve à l'extérieur.

Ces dépôts détritiques proviennent probablement de l'entrée de la cavité, exutoire jusqu'alors du système hydrologique des Monts Sarighayeh, suite à une inversion, peut être très épisodique, de l'écoulement hydrologique de surface. Cette inversion du sens de l'écoulement peut sans doute se comprendre à travers des mouvements orogéniques du Zagros entraînant un basculement régional de la topographie (qui n'est pas nécessairement de grande ampleur). Le caractère grossier de certains éléments et la forte hétérométrie des galets constitutifs du conglomérat suggèrent un transport souterrain relativement modeste avec un dépôt relativement brutal. De plus, si ce remplissage provenait de l'amont du réseau souterrain, il est probable que des témoins de ce remplissage seraient encore visibles dans différentes parties de la cavité.

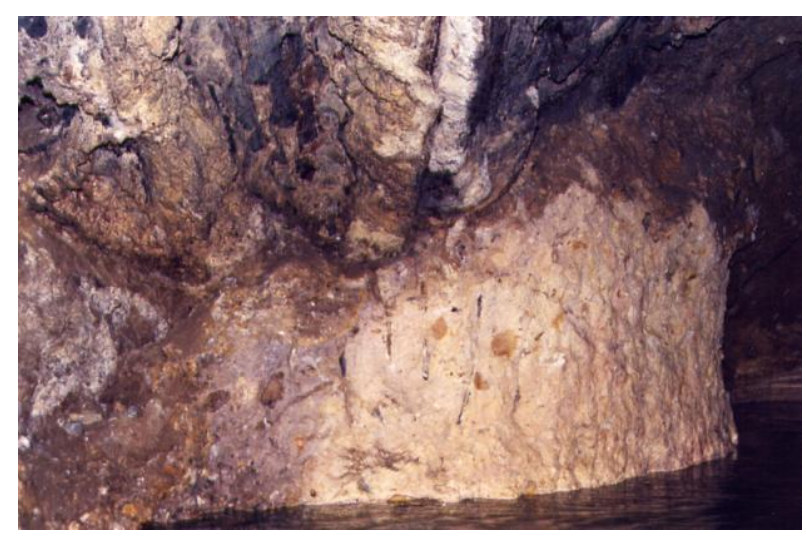

Photo 5. Le témoin d'un ancien remplissage conglomératique, on observe des lambeaux de ces sédiments collés au plafond. 


\section{- Paléoniveaux de la nappe karstique}

La plupart des galeries du réseau présentent sur leurs parois un étagement de niveaux concrétionnés. La calcite s'est développée en collerettes et en bourrelets plus ou moins épais à partir des parois. On retrouve trois niveaux très bien marqués représentant trois paléoniveaux de la nappe karstique (figure 7). Mais, il en existe beaucoup plus situés notamment entre 3,3 $\mathrm{m}$ et $6 \mathrm{~m}$ au-dessus du lac (photo 6). La genèse de ces concrétions montre qu'il s'agit bien d'une nappe ou d'un lac souterrain sans écoulement d'eau. Ces différents niveaux traduisent un stationnement durable de la nappe pendant lequel le système hydrologique présente un équilibre (battement de la nappe très faible voire inexistant).
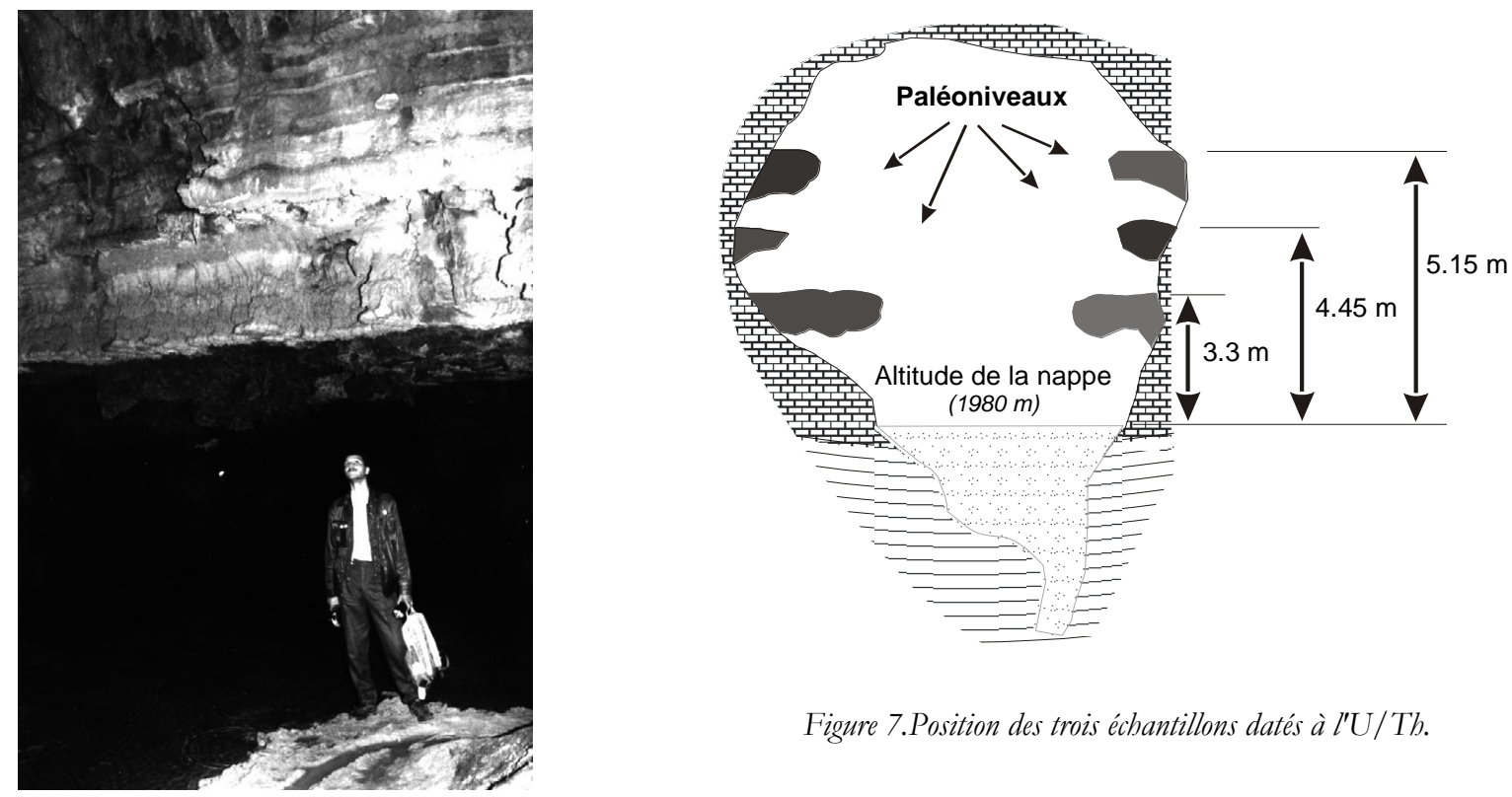

Figure 7.Position des trois échantillons datés à l'U/Th.

Photo 6. Les paléoniveaux de la nappe, le plus haut se situe à environ $6 \mathrm{~m}$ au dessus de la nappe.

Nous avons préciser l'âge de trois des paléoniveaux les plus remarquables que l'on suit sur la majorité des galeries dont nous avons mesuré précisément la position par rapport à la nappe (figure 7).

Tableau 1. Résultats des analyses uranium-thorium et âge des concrétions (datations U/Th, en 1996, au C.E.R.A.K., Faculté Polytechnique de Mons, Belgique, par Y. Quinif)

\begin{tabular}{lcccccc} 
Echantillon & $(U) \mathrm{ppm}$ & $234 \mathrm{U} / 238 \mathrm{U}$ & $230 \mathrm{Th} / 234 \mathrm{U}$ & $\mathbf{2 3 0} \mathrm{Th} / 232 \mathrm{Th} 234 \mathrm{U} / 238 \mathrm{U}$ à to Age (en m.a.) \\
\hline $3.3 \mathrm{~m}$ & $0.423( \pm 0.011)$ & $2.129( \pm 0.043)$ & $0.060( \pm 0.006)$ & 100 & 2.151 & $6.6(+0.7 /-0.6)$ \\
$4.45 \mathrm{~m}$ & $0.861( \pm 0.022)$ & $1.083( \pm 0.023)$ & $2.463( \pm 0.080)$ & $137 \pm 14$ & $\ldots$ & $\ldots$ \\
$5.15 \mathrm{~m}$ & $0.936( \pm 0.024)$ & $1.105( \pm 0.022)$ & $1.864( \pm 0.056)$ & $81 \pm 6$ & $\ldots$ & $\ldots$
\end{tabular}

Les erreurs indiquées entre parenthèses proviennent de la nature statistique des mesures dans un intervalle bilatéral de $67 \%$ (soit \pm un écart-type, pour une variable qui suit une loi normale).

Le trottoir calcitique du premier niveau, placé à $3.3 \mathrm{~m}$ au-dessus de la nappe, qui correspond aussi à la formation des pseudo-gours de plafond, est Holocène (tableau 1). Il se serait formé il y a environ 6.600 BP (tableau 1).

On ne peut éliminer parmi les causes qui ont entraîné l'abaissement successif et régulier du lac souterrain, une variation tectonique du niveau de base régional. Un rabattement de la nappe de 3,3 $\mathrm{m}$ depuis 6600 ans pourrait alors signifier une surrection régionale moyenne durant cette période d'environ $0,5 \mathrm{~mm} / \mathrm{an}$. Cependant, la genèse du premier niveau coïncide précisément avec la période Atlantique en Europe, réputée pour son climat doux et humide.

Les deux autres niveaux, placés respectivement à $4.45 \mathrm{~m}$ et $5.15 \mathrm{~m}$ au-dessus de la nappe, ont un rapport isotopique thorium 230 - uranium 230 supérieur à 1, ce qui implique l'ouverture géochimique du système (Quinif, 1989). 
Cependant le rapport thorium 230 - 232 est grand et démontre bien qu'il n'y a pas eu de contamination détritique. Toutefois le rapport isotopique uranium 234 - 230 est proche de 1, ce qui suggérerait la grande ancienneté de ces deux échantillons si nous admettons que l'ouverture géochimique du système ne modifie pas ou peu ce rapport isotopique et que le rapport isotopique initial est de l'ordre de celui mesuré dans l'échantillon holocène (commentaire de Y. Quinif). L'âge de ces deux trottoirs serait alors de 1.5 à 2 millions d'années.

\section{L'EVOLUTION GEOMORPHOLOGIQUE}

\section{A. Les coulées volcaniques perchées}

On observe à l'ouest des Monts Sarighayeh des coulées volcaniques perchées qui ont scellé une partie importante du relief. L'érosion différentielle a abouti à l'inversion du relief. Les épanchements volcaniques sont aujourd'hui placés en position perchée par rapport aux plaines avoisinantes. La coulée la plus proche de la cavité d'Alisadr se situe à 7 $\mathrm{km}$ (figure 4). Large de $1 \mathrm{~km}$, elle s'étend sur environ $3 \mathrm{~km}$ de long. Une épaisseur de plus de $90 \mathrm{~m}$ de basalte repose sur les marbres jurassiques, le sommet de cet épanchement volcanique se place aux alentours de $2150 \mathrm{~m}$ d'altitude et, à son pied à $2060 \mathrm{~m}$ d'altitude, le village de Halan-e-Sofla s'est installé (photo 7). Dans l'axe de cette coulée, à $25 \mathrm{~km}$ environ au SW d'Alisadr, à côté du village de Baharlou, le Mont de Dashkasan est le cône d'un volcan qui correspond à l'une des phases éruptives qui a participé à l'édification de ces coulées (photo 8). Ce cône, culminant à 2190 m, a plus de $300 \mathrm{~m}$ de dénivelé.

Nous avons effectué une datation potassium-argon sur un échantillon dans la coulée basaltique d'Halan-e-Sofla, et une autre sur le cône volcanique de Dashkasan. Les analyses du rapport 40K/40Ar ont été effectuées à l'Institut Physique du Globe de Strasbourg par M. Montigny. L'estimation de ce rapport permet d'évaluer l'âge de la coulée à 10,6 Ma (B.P.), et fournit un âge de 10,5 Ma (B.P.) pour le matériel volcanique de Dashkasan.

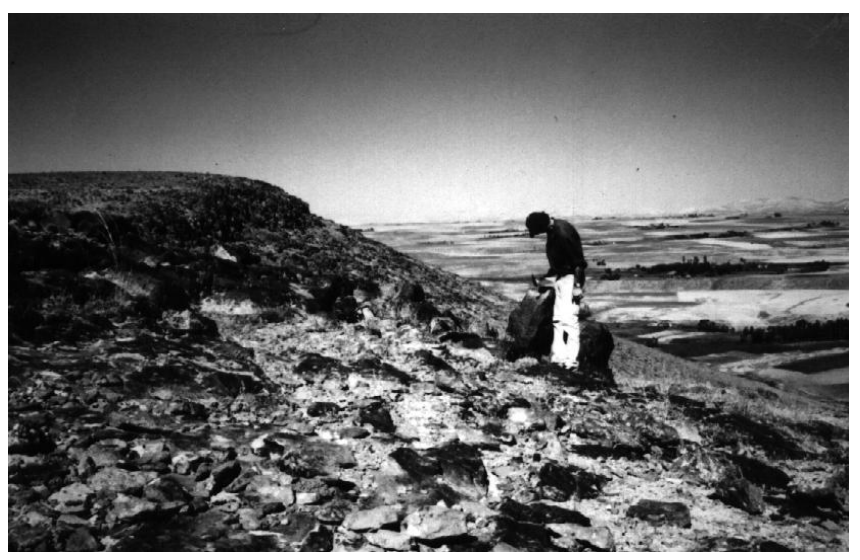

Photo 7. Coulée basaltique perchée de Halan-eSoflan. La grotte d'Alisadr se situe à droite de la photo. Une datation $40 \mathrm{~K} / 40 \mathrm{Ar}$ d'un échantillon de cette coulée situe cet épanchement basaltique au Miocène supérieur (10,6 Ma).

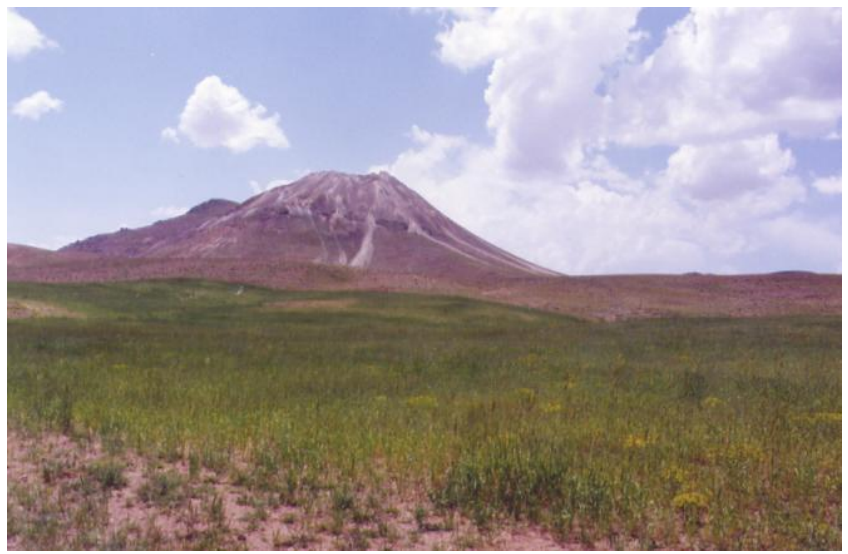

Photo 8. Volcan de Dashkasan

La convergence de l'âge des deux échantillons, d'origine pétrographique différente et avec des teneurs en potassium différentes, confirme une bonne appréciation de l'âge de ces deux phases volcaniques par cette méthode (tableau 2). Par ailleurs, l'étude du volcanisme dans la région de Bijar (au NE de Sanandaj) converge en partie avec ces résultats puisqu'elle montre deux phases : la première d'âge miocène supérieur et la seconde d'âge plio-quaternaire (Boccaletti 
et al., 1977, Baud, 1987 ; Moine-Vaziri et al., 1991). Ces résultats complètent ainsi notre connaissance du volcanisme du Zagros qui reste encore mal connu de nos jours.

Tableau 2. Résultats des datations $40 \mathrm{~K} / 40 \mathrm{Ar}$

(datation effectuées par M. Montigny à l'I.G.P. de Strasbourg)

\begin{tabular}{lccccc} 
Echantillons & $\begin{array}{c}\text { K20 } \\
(\%)\end{array}$ & $\begin{array}{c}\text { 40Ar* } \\
(\%)\end{array}$ & $\begin{array}{c}\text { 40Ar* } \\
(\mathbf{1 0 - 6 c c / g r})\end{array}$ & $\begin{array}{c}\text { Age } \\
\text { (M.a.) }\end{array}$ & $\begin{array}{c}\text { Erreur } \\
\text { (M.a.) }\end{array}$ \\
\hline Volcan de Dashkasan & 2.30 & 35.2 & 0.78 & $\mathbf{1 0 . 5}$ & 0.8 \\
Coulée basaltique & 10.00 & 23.0 & 3.44 & $\mathbf{1 0 . 6}$ & 0.9 \\
LP6 & 10.04 & 95.7 & 43.08 & 128.5 & 2.7
\end{tabular}

Précision sur les 3 derniers étalons $=1,2 \% ; 40 \mathrm{Ar}=40$ Ar radiogénique en unité STP; Erreur à $95 \% ;$ LP6 mesure sur un échantillon standard

\section{B. Evolution géomorphologique de la région et de la grotte d'Alisadr}

La région d'Alisadr permet ainsi de mieux appréhender l'évolution des régions du domaine métamorphique qui, sous l'influence de la montée progressive des massifs granitiques, sont soumises à l'érosion dès la fin du Paléogène (voire dès l'Oligocène),. Au début du Néogène, les formations conglomératiques recouvrant les marbres du Jurassique émergent peu à peu avec les formations mésozoïques (figure 8 : A). La partie méridionale de la région, la plus directement au contact avec le massif granitique d'Alvand (Valizadeh et al., 1975), se soulève plus rapidement, ce qui entraîne un basculement régional avec une faible pente en direction du nord. Un important réseau noyé se développe alors au cours du Néogène au niveau du contact entre les séries schisteuses imperméables et les marbres. Avec un débit probablement élevé, la source vauclusienne d'Alisadr permettait l'évacuation des eaux souterraines (figure $8:$ B). Ce système hydrologique karstique draine alors un bassin versant considérable qui comprend notamment les Monts Sarighayeh et les Monts Subashi. L'ensemble du réseau se termine au niveau de cette source par un entrelacs de conduits formant un "delta souterrain". Il existe encore de nombreuses traces de cette spéléogenèse en régime noyé dans les cavités d'Alisadr et de Sarab.

Le soulèvement se poursuit ensuite mais ne prend plus une ampleur importante. Cependant, l'émersion des nappes crétacées de la Crushed zone (Dumas, 1998) se répercute sur le domaine métamorphique par une compression de la couverture sédimentaire engendrant une série de cassures avec un rejet vertical de faible envergure (figure $8: \mathrm{C}$ ). Cette tectonique cassante est suffisante pour découper et compartimenter en un ensemble de gradins et de blocs les marbres et la couverture paléogène de la région. La connexion hydrologique du réseau endokarstique couvrant un large bassin versant est ainsi rompue. La source vauclusienne n'est plus active.

Sur ces gradins structuraux existe actuellement un ensemble de nappes plus ou moins perchées les unes par rapport aux autres et sans doute mises en interrelation par une fissuration du soubassement ou encore par de petits conduits noyés. L'étagement des aquifères karstiques est attesté par l'altitude des nappes karstiques aux deux extrémités des Monts Sarighayeh, elles se positionnent à $1980 \mathrm{~m}$ d'altitude dans la grotte d'Alisadr et à $2309 \mathrm{~m}$ d'altitude dans celle de Sarab. Par ailleurs, la présence de la source de Ghamish Li Gol, située à 2002 m, et de celle de Kouthal à 2024 m d'altitude montrent que des nappes intermédiaires existent au cœur des Monts Sarighayeh.

Rapidement, voire simultanément, s'effectue une érosion des sédiments détritiques sur les points hauts. Une partie du matériel issu du démantèlement de la couverture éocène est repris par un écoulement de surface venant se perdre dans l'ancienne source vauclusienne d'Alisadr. Un remplissage et un colmatage du réseau souterrain s'effectuent (figure $8: D$ ). Ce dernier force les eaux à circuler dans les parties hautes des galeries et favorise la genèse de nouvelles galeries latérales. Ce "bouchon" conglomératique est ensuite décapé en même temps qu'il est altéré. L'ablation s'effectue d'abord sur les parties hautes de la topographie (sur les Monts Sarighayeh et Subashi). Facilitée et préparée par des processus de bréchification, cette érosion ne touche pas uniquement la couverture détritique mais aussi une partie non négligeable des calcaires marbrifiés du Jurassique.

Il est probable que c'est lors de cette phase érosive que la coulée volcanique d'Halan-e-Sofla est mise en relief. Les datations de deux échantillons volcaniques montrent que cette érosion se place au moins après le Tortonien : l'âge de la coulée basaltique est de 10,6 Ma et celui du volcan à trachytes est de 10,5 Ma. L'ablation de la couverture tertiaire semble se situer à la fin du Miocène et au cours du Pliocène.

L'inexistence d'une érosion glaciaire et la pluviosité actuelle d'environ $300 \mathrm{~mm} /$ an (Atlas climatique d'Iran, 1965 ; Alijani et al., 1985 ; Dumas, 1998) rendent difficiles l'hypothèse d'une forte empreinte quaternaire sur les paysages. D'ailleurs, l'étude micromorphologique des sols, leur composition et leur texture attestent d'une pédogenèse relativement ancienne. Une observation microscopique de certains échantillons montre que ces sols renferment d'anciennes altérites et des paléosols qui n'ont pu se former que dans un contexte tropical chaud et humide. 


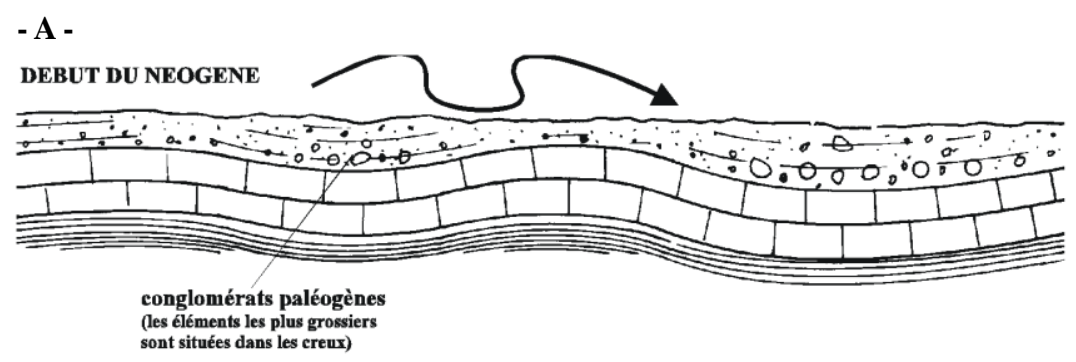

- B -

AU MIOCENE SUPERIEUR

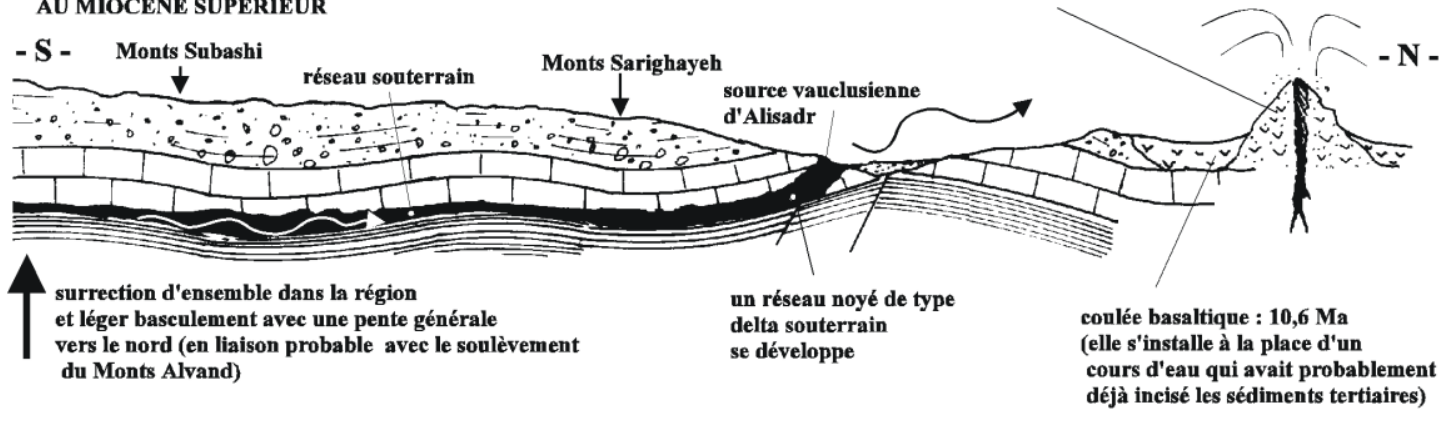

- C -

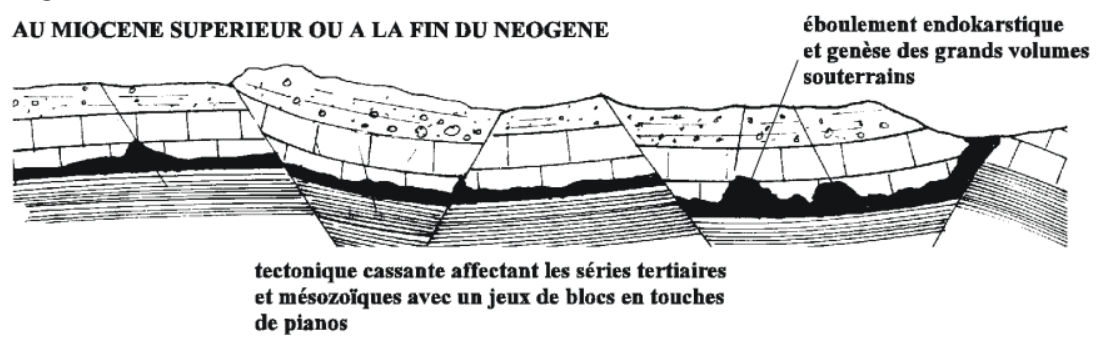

- D -

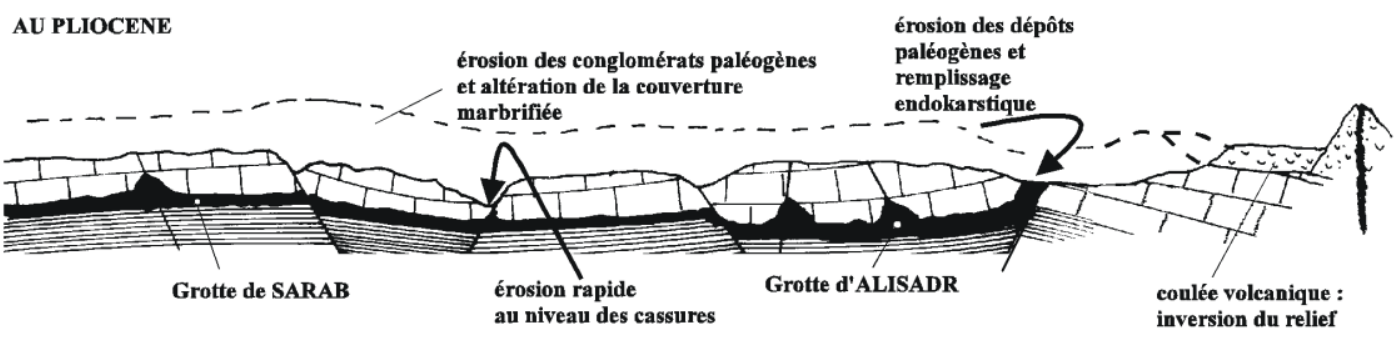

- E -

Au Quaternaire

ablation pléistocène

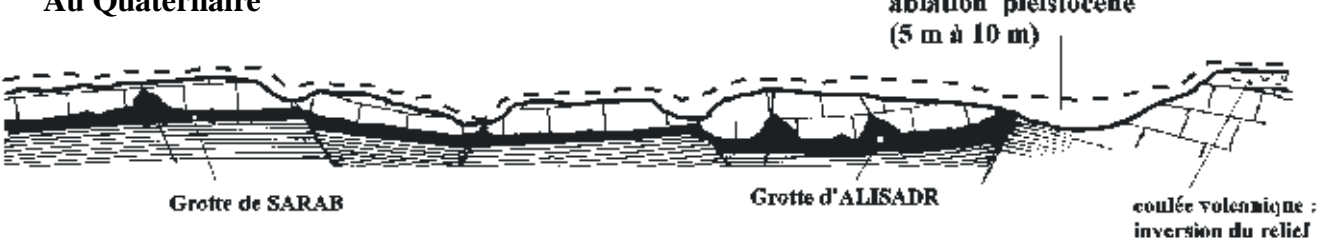

Figure 8. Evolution géomorphologique de la grotte d'Alisadr

L'ablation quaternaire pourrait avoir enlevé une tranche d'une dizaine de mètres d'épaisseur d'après la position perchée de l'ancienne source vauclusienne d'Alisadr. L'orifice de cette source est actuellement placée à 10-15 m audessus du niveau de base. Cette position pourrait être la conséquence d'une ablation moyenne de 5 à 7.5 $\mathrm{mm} /$ millénaire au cours du Quaternaire (figure $8: \mathrm{E}$ ). Tout en restant très prudent, cette érosion moyenne est également approchée par la datation de deux échantillons volcaniques du Miocène supérieur (10,6 Ma et 10,5 Ma). 
Une partie de ces épanchements volcaniques est aujourd'hui perchée à environ $100 \mathrm{~m}$ au dessus de la plaine d'Alisadr. Ceci fait pourrait également témoigner d'une érosion moyenne depuis la fin du Miocène de l'ordre de 10 $\mathrm{mm} /$ millénaire $\left(10^{6} \cdot\left[100 \mathrm{~m} / 10.10^{6}\right]\right)$. Mais cette comparaison n'est qu'indicative dans la mesure où l'ablation depuis la fin du Miocène recouvre une érosion chimique (les séries tertiaires sont carbonatées) et très probablement aussi une érosion mécanique. Enfin, cette très faible vitesse d'érosion converge avec la mesure de la dissolution spécifique actuelle d'environ $3 \mathrm{~mm} /$ millénaire (Dumas, 1998).

\section{CONCLUSION}

La chaîne du Zagros présente une karstification importante sur l'ensemble de ses massifs (Judson, 1973 ; Farcy, 1974 et 1975 ; Maire, 1990). Le domaine métamorphique du Zagros et ses hauts plateaux présentent une karstification complexe qui ne peut s'appréhender qu'à travers une évolution suffisamment longue des paysages. Différents indices paléoenvironnementaux conservés par le karst des Monts Sarighayeh, ainsi que la coulée d'Halan-e-Sofla, permettent de comprendre un peu mieux et retracer l'évolution de la cavité d'Alisadr. A partir de ces témoins, on a pu reconnaitre plusieurs phases morphogéniques et les positionner assez bien dans la chronologie géologique. Il apparaît plausible que les grands traits du paysage exokarstique et endokarstique soit pour l'essentiel terminée au Néogène. Cette hypothèse milite en faveur d'un soulèvement relativement modeste de la région au cours du Plio-Quaternaire, qu'il faudra confirmer par des travaux complémentaires.

Je remercie tout particulièrement M. Mietton et R. Maire pour leurs conseils. MM Akabary et Chabery, Directeurs de la cavité touristique d'Alisadr, pour m'avoir accueilli. M. Chardon pour sa relecture critique.

\section{Références}

Alijani B., Harman J.R., 1985. Synoptic Climatology of Precipitation in Iran. Annals of the Association of Americain Geographers, 75 (3), pp. 404-416.

Atlas climatique de l'Iran, 1965. Université de Téhéran. Sous la direction de Mostofi A. et avec la collaboration de Najaf-Abadi N., Ganji B.A. 117 planches climatiques.

Boccaletti M., Innocenti F., Manetti P., Mazzuoli R., Motamed A., Pasquare G., Radicati Di Brozolo F., Sobhani E., 1977. Neogene and Quaternary Volcanism of the Bijar Area. Bull. Volcanol., vol. 40-2, pp. 121-130.

Boccaletti M., Innocenti F., Manetti P., Mazzuoli R., Motamed A., Pasquare G., Radicati Di Brozolo F., Sobhani E., 1977. Neogene and Quaternary Volcanism of the Bijar Area. Bull. Volcanol., vol. 40-2, pp. 121-130.

Braud J., 1987. La suture du Zagros au niveau de Kermanshah (Kurdistan iranien) : reconstitution paléogéographique, évolution géodynamique, magmatique et structurale. Thèse d'Etat, Université de ParisSud, p. 489.

Dumas D., 1998 : Karsts du Zagros (Iran). Bilans hydrologiques et évolution géomorphologique. Thèse Univ. Louis Pasteur Strasbourg, CEREG, 460 pages.

Dumas D., Mietton M., Maire R., 1994. La grotte aménagée d'Ali Sadr (Iran). Karstologia n²3, 60-63.

Farcy J.-P., 1974. Expéditions hauts karsts iraniens. Spelunca n4, pp. 107-108.

Farcy J.-P., 1975. Troisième expédition spéléologique française en Iran. Rapport du Centre Niçois d'Expéditions Spéléologiques, Féd. Fr. de Spéléologie, p. 26.

Goodchild M.F., Ford D.C., 1971. Analysis of scallops pattern by simulaion under controlled conditions. J. of Geology, 79, pp. 52-62.

Judson D., 1973. Ghar Parau. Ed Cassell, London, p. 216.

Judson D., 1973. The discovery and exploration of Ghar Parau (Iran). Trans. Cave Research Group of Great Britain, vol. $15, \mathrm{n}^{\circ} 1$, pp. 19-26.

Lismonde B., Lagmani A., 1987. Les vagues d'érosion. Karstologia, n¹0, pp. 33-38.

Maire R., 1990. La haute montagne calcaire. Karstologia Mémoires, n³, p. 731.

Moine-Vaziri H., Khalili-Marandi S., 1991. Importance d'un volcanisme potassique, au miocène supérieur, en Azerbaidjan (Iran). C. R. Acad. Sc. Paris, t. 313, série II, pp. 1603-1610.

Nicod J., 1996. Karst et mines en France et en Europe : gîtes, grottes-mines et géotechnique. Karstologia, n²7, pp. $1-20$.

Quinif Y., 1989. La datation uranium-thorium. Spéléochronos $n^{\circ} 1$. Centre d'études et de recherches appliquées au karst. Mons, Belgique. pp. 3-22.

Ricou L.E., 1976. Evolution structurale des Zagrides. La région clef de Neyriz (Zagros iranien). Mém. de la Soc. Géol. de Francz, n¹5, p. 137.

Valizadeh M.V. et Cantagrel J.M., 1975. Premières données radiométriques (K/Ar et Rb/Sr) sur les micas du complexe magmatique du Mont Alvand, près de Hamadan (Iran occidental). C. R. Acad. Sc. Paris, t.281, D, pp. 1083-1086. 